\title{
OUTCOME AFTER STEROID WITHDRAWAL IN PEDIATRIC RENAL TRANSPLANT PATIENTS RECEIVING TACROLIMUS-BASED IMMUNOSUPPRESSION 1
}

\author{
Pradip Chakrabarti $^{2}$, Hsien Y. Wong ${ }^{2}$, Velma P. Scantlebury ${ }^{2}$, Mark L. Jordan ${ }^{3}$, Carlos \\ Vivas $^{3}$, Demetrius Ellis ${ }^{4}$, Susan Lombardozzi-Lane ${ }^{4}$, Thomas R. Hakala ${ }^{3}$, John J. Fung ${ }^{2}$, \\ Richard L. Simmons ${ }^{5}$, Thomas E. Starzl ${ }^{2}$, and Ron Shapiro 2,6 \\ Thomas E. Starzl Transplantation Institute, Division of Urologic Surgery, Pediatric Nephrology, and \\ Division of General and Vascular Surgery, University of Pittsburgh, Pittsburgh, Pennsylvania
}

\begin{abstract}
Background-Corticosteroids have always been an integral part of immunosuppressive regimens in renal transplantation. The primary goal of this analysis was to assess the safety of steroid withdrawal in our pediatric renal transplant recipients receiving tacrolimus-based immunosuppression.
\end{abstract}

\begin{abstract}
Methods-Between December 1989 and December 1996, 82 renal transplantations were performed in pediatric patients receiving tacrolimus-based immunosuppression. Two of these patients lost their grafts within 3 weeks of transplantation (and were still on steroids at the time of graft loss), and were excluded from further analysis. Seventy-four patients $(92.5 \%)$ were taken off prednisone a median of 5.7 months after transplantation. Of these 74, $56(70 \%)$ remained off prednisone (OFF), and 18 $(22.5 \%)$ were restarted on prednisone a median of 14.8 months after discontinuing steroids $(\mathrm{OFF} \rightarrow$ $\mathrm{ON})$. 6(7.5\%) were never taken-off prednisone $(\mathrm{ON})$. The mean follow-up was $59 \pm 23$ months.
\end{abstract}

Results-The 1-, 3-, and 5-year actuarial patient survival rates in the OFF group were 100\%, 98\%, and $96 \%$, respectively; in the OFF $\rightarrow$ ON group, they were $100 \%, 100 \%$, and $100 \%$, and in the ON group, they were $100 \%, 83 \%$, and $83 \%$. The 1-, 3-, and 5- year actuarial graft survival rates in the OFF group were $100 \%, 95 \%$, and $82 \%$, respectively; in the OFF $\rightarrow$ ON group, they were $100 \%$, $89 \%$, and $83 \%$; and in the ON group, they were $100 \%, 50 \%$, and $33 \%$. Two of the six graft losses in the OFF group, three out of four in the OFF $\rightarrow$ ON Group, and two out of five in the ON group, were to chronic rejection. A time-dependent Cox regression analysis showed that the hazard for graft failure for those who came and stayed off prednisone was 0.178 relative to those who were never withdrawn from prednisone $(P=0.005)$. Patients who were 10 years of age or younger were withdrawn from prednisone earlier (median: 5 months) than those older than 10 years (median: 7.3 months, $P=0.02$ ). In addition, patients who never had acute rejection were withdrawn from steroids earlier (median: 5 months) than those who had one or more episodes of acute rejection (median: 7.6 months, $P=0.001)$. There was no effect of donor age, race, sex, recipient race, sex, cadaveric versus living donor, 48-hr graft function, panel reactive antibody, and total HLA mismatches or matches on the likelihood of being weaned off steroids. Serum creatinine at most recent follow-up in the OFF group was $1.2 \pm 0.5 \mathrm{mg} / \mathrm{dl}$; in the $\mathrm{OFF} \rightarrow \mathrm{ON}$ group, it was $1.8 \pm 0.9 \mathrm{mg} / \mathrm{dl}$, and in the $\mathrm{ON}$ group it was 2.0

\footnotetext{
${ }^{1}$ Presented at the 18th Annual Meeting of the American Society for Transplantation, May 15-19, 1999, Chicago, IL.

${ }^{6}$ Address reprint requests to: Ron Shapiro, M.D., 4th Floor Falk, 3601 Fifth Avenue, Pittsburgh, PA 15213.

2 Thomas E. Starzl Transplantation Institute.

${ }^{3}$ Division of Urologic Surgery.

${ }_{5}^{4}$ Pediatric Nephrology.

${ }^{5}$ Division of General and Vascular Surgery.
} 
$\mathrm{mg} / \mathrm{dl}(P<0.003)$. The incidence of rejection in the OFF, OFF $\rightarrow$ ON, and ON groups was $39 \%, 77 \%$, and $100 \%$, respectively $(P<0.05)$.

Conclusion-These data suggest that steroid withdrawal in pediatric renal transplant patients receiving tacrolimus-based immunosuppression is associated with reasonable short- and mediumterm patient and graft survival, and acceptable renal function. Patients who discontinue and then resume steroids had patient and graft survival rates comparable with those in patients who discontinue and stay off steroids, but had a higher serum creatinine and a higher incidence of rejection.

In spite of the well-established side effects associated with their use (1), corticosteroids have remained a part of long-term immunosuppression after renal transplantation. With the introduction of tacrolimus, however, steroid withdrawal has been possible in some $70 \%$ of successfully transplanted adults and children (2-9). In the latter group, significant catch-up growth has been achieved, particularly in preadolescent children $(5,6)$. An important question, however, concerns the outcome after steroid withdrawal in pediatric patients on tacrolimus. Although this question has been studied in adults $(4,7-9)$, it has not been investigated in detail in children. We looked at our pediatric renal transplant patients receiving tacrolimus-based immunosuppression and examined the short- and medium-term outcomes after steroid withdrawal.

\section{PATIENTS AND METHODS}

Between December 14, 1989, and December 31, 1996, 82 renal transplantations under tacrolimus-based immunosuppression were performed in 81 pediatric patients at the Children's Hospital of Pittsburgh (Table 1). Two grafts were lost within 3 weeks of transplantation and were excluded from further analysis (the recipients were still on steroids at the time of graft loss). The mean recipient age was $10.5 \pm 5.1$ years (range: $0.7-17.9)$. Sixty-four (80\%) were undergoing their first transplant, and $16(20 \%)$ were undergoing their second $(\mathrm{n}=10)$, third $(\mathrm{n}=5)$, or fourth $(\mathrm{n}=1)$ transplants. Five (6\%) had panel-reactive antibody (PRA) levels of $40 \%$ or higher, and all of them were undergoing repeat transplantation. There were $68(85 \%)$ Caucasian, 7 (9\%) African-American, and $5(6 \%)$ Asian recipients. The causes of end-stage renal disease are listed in Table 2.

The mean donor age was $27.9 \pm 14.5$ years (range: $0.7-50$ ). Forty-six (58\%) kidneys were from cadaveric donors. The mean cold ischemia time was $27.5 \pm 8.8 \mathrm{hr}$ (range: 9.3-45.2). There were $34(42 \%)$ living donors. The mean number of HLA matches and mismatches was $2.9 \pm 1.3$ and $3.0 \pm 1.3$, respectively. There were four (5\%) 0 -antigen mismatch cases and one (1\%) HLAidentical sibling donor case.

Immunosuppression was with tacrolimus and steroids as previously described $(5,6)$. Induction antilymphocyte antibody was not used. Eighteen patients (22\%) received azathioprine. Mycophenolate mofetil was not used as an initial immunosuppressive agent. The idealized timetable for steroid withdrawal is shown in Table 3. Although there were no formal a priori inclusion or exclusion criteria for steroid withdrawal, patients with multiple rejection episodes were unlikely to be candidates for complete withdrawal. Steroids were tapered and discontinued in patients who maintained stable allograft function as the steroid dosage was gradually decreased. Steroids were restarted in recipients who experienced a rising serum creatinine with biopsy-proven acute rejection. Allograft failure was defined by return to dialysis, repeat transplantation, or death.

Patient survival was calculated from the date of kidney transplantation until death, and graft survival was calculated from the date of kidney transplantation until graft failure or patient death. Survival curves were generated using the Kaplan-Meier (product limit) method and were compared using the log-rank test. Values are presented as mean \pm SD. A time-dependent Cox 
regression analysis for hazard assumption was done to compare graft survival in recipients who came off steroids versus those who never came off. A Cox time-dependent covariate $z(t)$ was modeled, so that $z(t)=1$ if the patient was weaned off at least once, and $z(t)=0$ if the patient was not weaned off by time $t$.

A multivariate multiple logistic regression analysis was performed to compare the effect of recipient sex, recipient race, delayed graft function, recipient PRA, total HLA matches, total HLA mismatches, donor age, and donor type (cadaveric vs. living related) on steroid withdrawal. The means between groups were compared by one-way analysis of variance. Catagorical data were analyzed by Pearson's chi-square method. $P$-values less than 0.05 were considered statistically significant.

Until June 1994, when tacrolimus was approved by the United States Food and Drug Administration, all transplants were performed under a protocol approved by the Human Rights Committee of the Children's Hospital of Pittsburgh.

\section{RESULTS}

The mean follow-up was 59 \pm 23 months (range: $11-103)$. Seventy-four (92.5\%) patients were withdrawn from steroids at a median of 5.7 months after transplantation. Fifty-six (70\%) remained off steroids, and $18(22.5 \%)$ resumed steroids at median of 14.8 months after steroid withdrawal (of these 18, 7 [39\%] were eventually tapered off steroids again). Six (7.5\%) patients never came off steroids (Tables 4 and 5). The overall 1-, 3-, and 5-year actuarial patient and graft survival rates were $100 \%, 97 \%$, and $96 \%$, and $100 \%, 90 \%$, and $79 \%$, respectively (Fig. 1). The 1-, 3-, and 5-year actuarial patient and graft survival rates for those who came and stayed off steroids (OFF) was $100 \%, 98 \%$, and $96 \%$, and $100 \%, 95 \%$, and $82 \%$, respectively (Figs. 2 and 3). Those who came off and later resumed steroids (OFF $\rightarrow \mathrm{ON}$ ) had a 1-, 3-, and 5-year actuarial patient survival of $100 \%$, and a 1-, 3-, and 5-year actuarial graft survival of 100\%, 89\%, and 83\% (Figs. 2 and 3). In those who never came off steroids (ON), the 1-, 3-, and 5-year actuarial patient survival was $100 \%, 83 \%$, and $83 \%$, and the 1-, 3-, and 5 -year actuarial graft survival was $100 \%, 50 \%$, and $33 \%$ (Table 4, parts B and C, Figures 2 and 3). Three patients died, 1.3-3.2 years after transplantation. Two were off steroids; one died of pancreatitis, and one died on dialysis, 1.3 and 3.2 years after transplantation (the patient on dialysis had lost her kidney to noncompliance 1.7 years after transplantation). One patient who was on steroids died of fungal sepsis 1.3 years after transplantation. Fifteen patients lost their allografts 1.2-6.6 years after transplantation, to rejection $(n=7)$, recurrent disease $(n=5)$, pancreatitis $(n=1)$, fungal sepsis $(n=1)$, and noncompliance $(n=1)$. In the OFF group, six grafts were lost: two to rejection, two to recurrent disease, one to noncompliance, and one to pancreatitis. In the OFF $\rightarrow \mathrm{ON}$ group, four grafts were lost: three to rejection, and one to recurrent disease. In the $\mathrm{ON}$ group, five grafts were lost: two to rejection, two to recurrent disease, and one to fungal sepsis.

There was a higher percentage of patients undergoing repeat transplantation in the ON group $(\mathrm{n}=5 ; 83 \%)$ compared to OFF group $(\mathrm{n}=7 ; 12 \%)$, and $\mathrm{OFF} \rightarrow \mathrm{ON}$ group $(\mathrm{n}=4 ; 22 \%)(P=0.001)$. The mean PRA was $34.5 \pm 38$ for the ON group, $5.6 \pm 15(P=0.001)$ for the OFF group, and 4.2 \pm 10 for the OFF $\rightarrow$ ON group $(P=0.002)$. A total of $39 \%$ of patients in the OFF group had experienced acute rejection before steroid withdrawal. In the $\mathrm{OFF} \rightarrow \mathrm{ON}$ group, the incidence of acute rejection was $77 \%$ before steroid withdrawal, and $100 \%$ after steroid withdrawal. In the ON group, rejection was seen in $100 \%$ of patients $(P<0.05)$ (Table 5). In recipients with functioning allografts, the mean serum creatinine at most recent follow-up in the OFF group was $1.2 \pm 0.5 \mathrm{mg} / \mathrm{dl}, 1.8 \pm 0.9 \mathrm{mg} / \mathrm{dl}$ in the $\mathrm{OFF} \rightarrow \mathrm{ON}$ group, and $2.0 \mathrm{mg} / \mathrm{dl}$ in the one patient with a functioning kidney in the $\mathrm{ON}$ group $(P=0.003)$. The mean tacrolimus level was $6.3 \pm 2.3$ $\mathrm{ng} / \mathrm{ml}$ in the OFF group, $6.5 \pm 2.5 \mathrm{ng} / \mathrm{ml}$ in the $\mathrm{OFF} \rightarrow \mathrm{ON}$ group, and $13.3 \mathrm{ng} / \mathrm{ml}$ in the $\mathrm{ON}$ 
group. At most recent follow-up in recipients with functioning allografts, $77 \%$ of the patients in the OFF group were off antihypertensive medications, compared with $44 \%$ in the OFF $\rightarrow$ $\mathrm{ON}$ group, and $0 \%$ in the $\mathrm{ON}$ group (Table $6 ; P=0.05$ ). There were no statistically significant differences in the hematocrit, white blood cells, platelets, cholesterol and triglyceride levels, and systolic and diastolic blood pressures among the three groups (Table 6).

In the multivariate analysis, patients who were 10 years of age or younger were withdrawn from prednisone earlier (median: 5 months) than those older than 10 years of age (median: 7.3 months, $P=0.02$ ). By 2 years after transplantation, $95 \%$ of patients 10 years old or younger were weaned off steroids, whereas $90 \%$ of patients older than 10 were weaned off steroids. If the patients were not weaned off steroids within the first 2 years, they were unlikely to be weaned off steroids at all. In addition, patients who never had acute rejection were withdrawn from steroids earlier (median: 5 months) than those who had one or more episodes of acute rejection (median: 7.6 months, $P=0.001$ ). By 2 years after transplantation, $100 \%$ of those without an episode of rejection were weaned off steroids, whereas $85 \%$ of those with at least one episode of rejection were weaned off steroids. Finally, there were no significant effect of donor age, race, sex, recipient race, sex, cadaveric vs. living related donor, 48-hr graft function, panel reactive antibodies, degree of HLA matching or mismatching, or immunosuppressive regiment (FK+steroids vs. FK+azathioprine+steroids) on the probability of being weaned off steroids.

\section{DISCUSSION}

Corticosteroids have essentially always been an integral part of immunosuppressive regimens in renal transplantation. In order to minimize their well-described side effects, there have been a number of attempts to reduce the dosage of corticosteroids, particularly after the advent of cyclosporine, when further reduction in the maintenance dosage was possible. Several trials have been undertaken to evaluate the outcome of corticosteroid withdrawal in patients receiving cyclosporine-based immunosuppression, with, in the end, not completely satisfactory results and an unacceptably high incidence of late rejection (9-19). Most renal transplant patients still receive maintenance corticosteroids chronically.

The primary goal of this analysis was to assess the safety of steroid withdrawal in our pediatric renal transplant recipients receiving tacrolimus-based immunosuppression. Medium-term outcomes suggest that steroid withdrawal was associated with acceptable patient and graft survival and stable renal function. Long-term steroid withdrawal was possible in $70 \%$ of patients, with 5-year actuarial patient and graft survival rates of $96 \%$ and $82 \%$, an incidence of rejection of $39 \%$, and a mean serum creatinine level of $1.2 \pm 0.5 \mathrm{mg} / \mathrm{dl}$. In the $22.5 \%$ of patients in whom steroids were withdrawn and then resumed (39\% of whom eventually came back off steroids), the 5-year actuarial patient and graft survival rates were $100 \%$ and $83 \%$, the incidence of rejection was $77 \%$, and the serum creatinine level was $1.8 \pm 0.9 \mathrm{mg} / \mathrm{dl}$. In the small group of patients $(7.5 \%)$ who never came off steroids, the 5-year actuarial patient and graft survival rates were $83 \%$ and $33 \%$, the incidence of rejection was $100 \%$, and the serum creatinine level was $2.0 \mathrm{mg} / \mathrm{dl}$ in the remaining patient with a functioning renal allograft. In this latter group, the percentage of patients undergoing repeat transplantation was higher (83\%; $P=0.001)$, as was the mean PRA $(34.5 \% \pm 38.5 \% ; P=0.001)$, than those in the OFF and OFF $\rightarrow$ ON groups.

It should be emphasized that patients were not randomly assigned to steroid withdrawal, and patients who never came off steroids had a number of risk factors, including repeat transplantation, sensitization, and acute rejection. The main questions for us concerned the safety of steroid withdrawal and, perhaps more importantly, the outcomes in patients who were withdrawn from and then resumed steroids. Five-year patient and graft survival rates were 
essentially identical in the OFF and the OFF $\rightarrow$ ON groups, but the incidence of rejection and the mean serum creatinine were higher in the OFF $\rightarrow$ ON group, and these findings are of concern with regard to longer-term follow-up. We have previously reported on the benefits of steroid withdrawal in children, including significant catch-up growth, a low incidence of hypertension, and normal cholesterol levels $(5,6)$. Unfortunately, there are no useful markers to identify patients who can be safely withdrawn from steroids.

To summarize, long-term steroid withdrawal in pediatric renal transplant patients receiving tacrolimus-based immunosuppression was associated with reasonable long-term patient and graft survival and renal function. Patients who discontinued and then resumed steroids had comparable patient and graft survival rates, but a higher incidence of rejection and higher serum creatinine levels. Patients who never came off steroids had the worst outcomes. In order to identify which pediatric renal transplant recipients are most likely to benefit from steroid withdrawal, prospective, randomized trials with well-defined criteria will likely be necessary.

\section{Acknowledgments}

The authors thank Gerri James, R.N., C.C.T.C.; Jennifer Lignoski, R.N., B.S.N., C.C.T.C.; M.J. Grosso, R.N., M.S.N.; Deborah Good, R.N., B.S.N., C.C.T.C.; Holly Woods, R.N., C.C.T.C.; Jareen Flohr, R.N., B.S.N., C.C.T.C.; Sue Bauder, R.N., B.S.N., C.C.T.C.; Sharon Orlofske, R.N, C.C.T.C.; and Mark Paynter, R.N., B.S.N., for their help with patient care; Sheila Fedorek, R.N., C.C.R.C.; Cynthia Eubanks; and Lynn Ostrowski, R.N, B.S.N., for help with data collection; and Amanda Gregan, B.A., for help with typing the manuscript and table preparation.

\section{References}

1. Fung, JJ. Conventional immunosuppressive agents. In: Shapiro, R.; Simmons, RL.; Starzl, TE., editors. Renal transplantation. Stamford, CT: Appleton \& Lange; 1997. p. 183

2. Shapiro R, Jordan ML, Scantlebury VP, et al. Tacrolimus in renal transplantation. Transplant Proc 1996;28(4):2117. [PubMed: 8769173]

3. Shapiro R. Tacrolimus (FK-506) in kidney transplantation. Transplant Proc 1997;29:45. [PubMed: 9123077]

4. Shapiro R, Jordan ML, Scantlebury VP, et al. Outcome after steroid withdrawal in renal transplant patients receiving tacrolimus-based immunosuppression. Transplant Proc 1998;30:1375. [PubMed: 9636557]

5. Shapiro R, Scantlebury VP, Jordan ML, et al. Pediatric renal transplantation under tacrolimus-based immunosuppression. Transplantation 1999;67(2):299. [PubMed: 10075598]

6. Shapiro R, Scantlebury VP, Jordan ML, et al. Tacrolimus in pediatric renal transplantation. Transplantation 1996;62(12):1752. [PubMed: 8990356]

7. Grewal HP, Thistlethwaite JR Jr, Loss GE, et al. Corticosteroid cessation 1 week following renal transplantation using tacrolimus/mycophenolate mofetil based immunosuppression. Transplant Proc 1998;30:1378. [PubMed: 9636558]

8. Cronin DC II, Bruce DS, Newell MA, et al. Tacrolimus therapy for refractory renal allograft rejection: experience with steroid withdrawal. Transplant Proc 1997;29(1-2):307. [PubMed: 9123010]

9. Woodle ES. Corticosteroid withdrawal in renal transplantation. Transplant Proc 1999;31:247. [PubMed: 10083094]

10. StSinclair NR. Low-dose steroid therapy in cyclosporine-treated renal transplant recipients with wellfunctioning grafts. Can Med Assoc J 1992;147(5):645. [PubMed: 1521210]

11. Ratcliffe PJ, Dudley CRK, Higgins RM, et al. Randomised controlled trial of steroid withdrawal in renal transplant recipients receiving triple immunosuppression. Lancet 1996;348:643. [PubMed: 8782754]

12. Ghandour FZ, Knauss TC, Mulligan DC, et al. Influence of steroid withdrawal on proteinuria in renal allograft recipients. Clin Transplant 1997;11:395. [PubMed: 9361929] 
13. Beik AI, Higgins RM, Lam FT, Morris AG. Steroid withdrawal and donor-specific hyporeactivity after cadaveric renal allotransplantation on maintenance triple therapy. Nephrol Dial Transplant 1997;12:1949. [PubMed: 9306348]

14. Hricik DE, Whalen CC, Lautman J, et al. Withdrawal of steroids after renal transplantation: clinical predictors of outcome. Transplantation 1992;53(1):41. [PubMed: 1733083]

15. Kupin W, Venkat KK, Heung K, et al. Complete replacement of methylprednisolone by azathioprine in cyclosporine-treated primary cadaveric renal transplant recipients. Transplantation 1998;45:53. [PubMed: 3276062]

16. Hricik DE, Bartucci MR, Mayes JT, et al. The effects of steroid withdrawal on the lipoprotein profiles of cyclosporine-treated kidney and kidney-pancreas transplant recipients. Transplantation 1992;54 (5):868. [PubMed: 1440855]

17. Hricik DE, Bartucci MR, Moir EJ, et al. Effects of steroid withdrawal on posttransplant diabetes mellitus in cyclosporine-treated renal transplant recipients. Transplantation 1991;51:374. [PubMed: 1994531]

18. Pirsch JD, Armburst MJ, Knechtle SJ, et al. Effects of steroid withdrawal on hypertention and cholesterol levels in living related recipients. Transplant Proc 1991;23:1363. [PubMed: 1989238]

19. Hricik DE, Lautman J, Bartucci MR, et al. Variable effects of steroid withdrawal on blood pressure reduction in cyclosporine-treated renal transplant recipients. Transplantation 1992;53(6):1232. [PubMed: 1604477] 
Kaplan-Meier Overall Sunvival

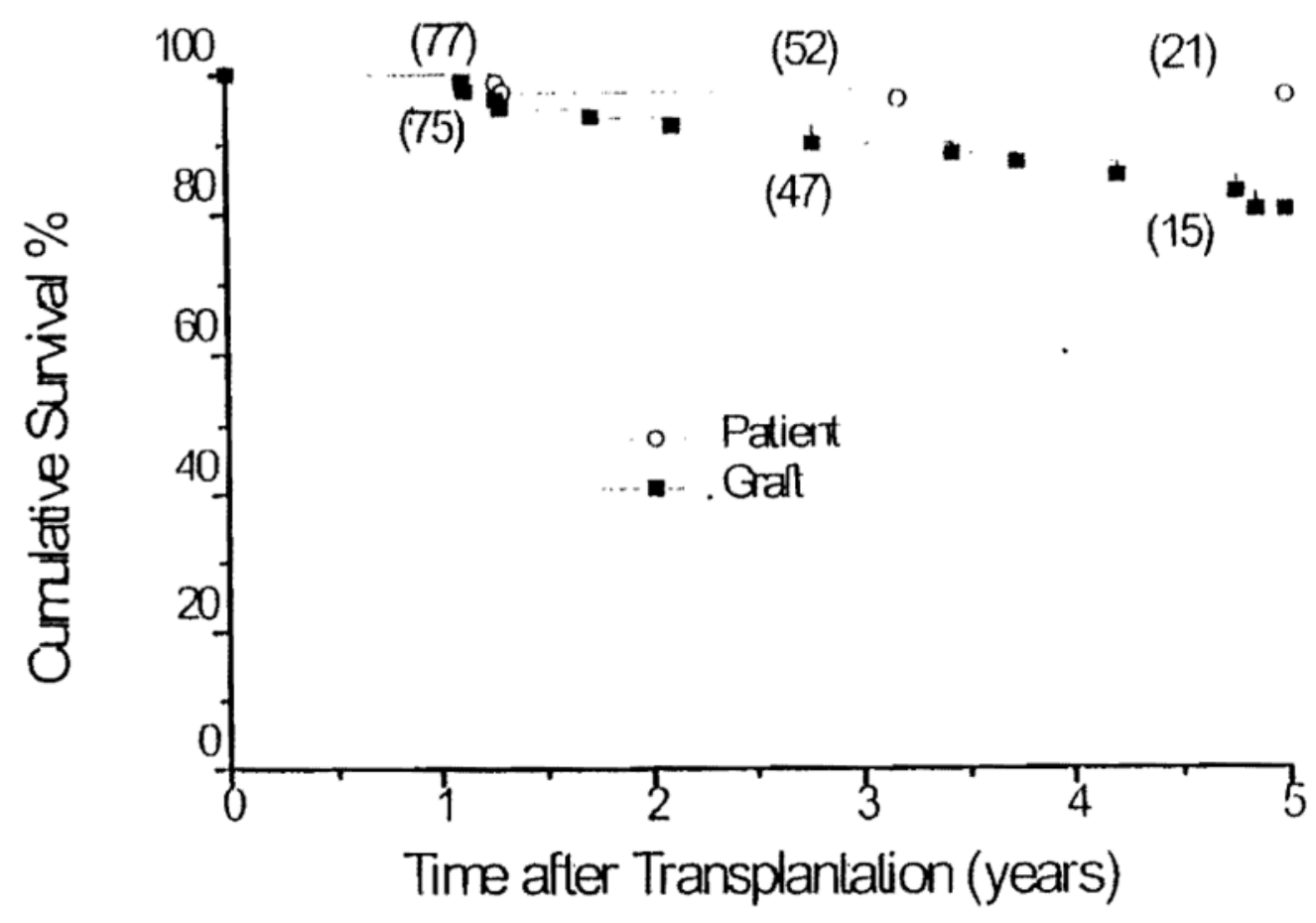

Figure 1.

Overall actuarial patient and graft survival. 
Kaplan-Meier: Patient Survival

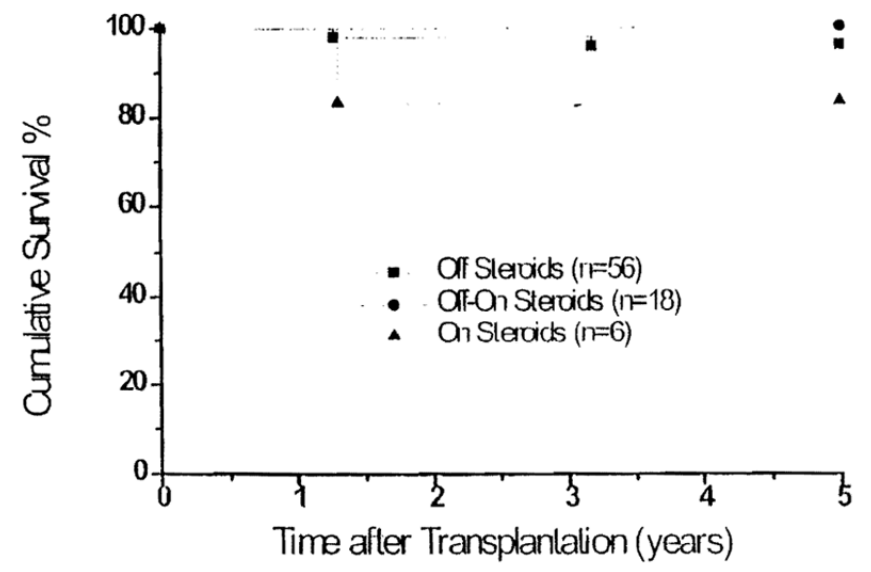

Figure 2.

Actuarial 1-, 3-, and 5-year patient survival in those off steroids, those off and then back on steroids, and those never off steroids. 


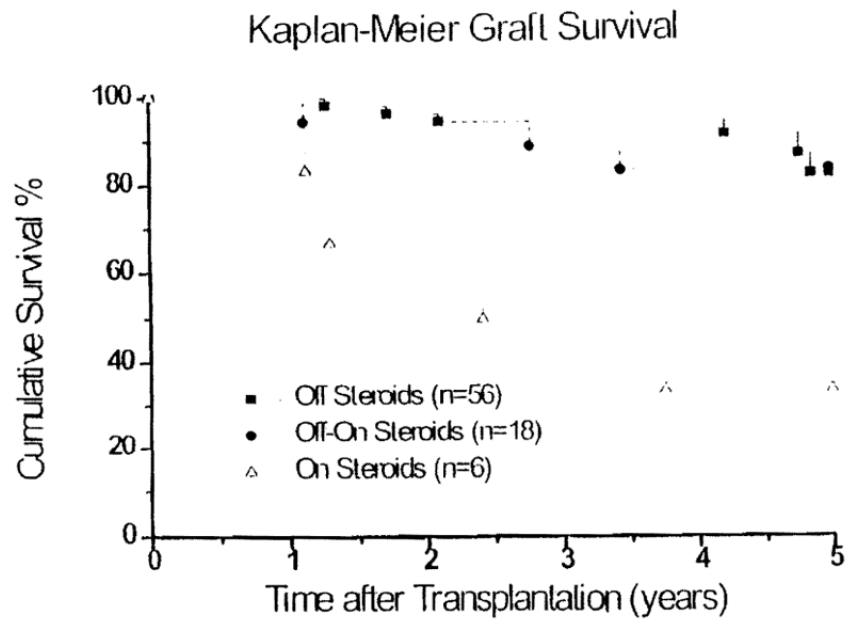

Figure 3.

Actuarial 1-, 3-, and 5-year graft survival in those off steroids, those off and then back on steroids, and those never off steroids. 


\section{Table 1}

Recipient/donor demographics

\begin{tabular}{lc}
\hline $\mathrm{n}$ & 80 \\
Recipient age (yr) & $10.5 \pm 5.1$ (range: $0.7-17.9)$ \\
First transplant & $64(80 \%)$ \\
Repeat transplants & $16(20 \%)$ \\
Second transplant & $10(13 \%)$ \\
Third transplant & $5(6 \%)$ \\
Fourth transplant & $1(1 \%)$ \\
PRA $\geq 40 \%$ & $5(6 \%)$ \\
PRA<40\% & $75(94 \%)$ \\
Donor age (yr) & $27.9 \pm 14.5($ range: $0.7-50)$ \\
Living donor & $34(42 \%)$ \\
Cadaveric donor & $46(58 \%)$ \\
Cold ischemia time (hr) & $27.5 \pm 8.8($ range: $9.3-45.2)$ \\
HLA & \\
Match & $2.9 \pm 1.3$ \\
Mismatch & $3.0 \pm 1.3$ \\
0-Ag mismatch & $4(5 \%)$ \\
HLA-identical LRD & $1(1 \%)$ \\
\hline
\end{tabular}

Transplantation. Author manuscript; available in PMC 2010 November 8. 


\section{Table 2}

Causes of end-stage renal disease

\begin{tabular}{lc}
\hline Obstructive uropathy & $14(17 \%)$ \\
Congenital dysplasia & $12(15 \%)$ \\
Membranoproliferative glomerulonephritis & $8(10 \%)$ \\
Focal segmental glomerulosclerosis & $7(9 \%)$ \\
Polycystic kidney disease & $4(5 \%)$ \\
Hemolytic-uremic syndrome & $3(4 \%)$ \\
Prune belly syndrome & $3(4 \%)$ \\
Congenital hypoplasia & $3(4 \%)$ \\
Ureteral reflux & $3(4 \%)$ \\
Chronic glomerulonephritis & $2(2 \%)$ \\
Interstitial nephritis & $2(2 \%)$ \\
Pyelonephritis & $2(2 \%)$ \\
Alport's syndrome & $2(2 \%)$ \\
Cystinosis & $2(2 \%)$ \\
Hereditary nephritis & $2(2 \%)$ \\
Undetermined & $11(14 \%)$ \\
\hline
\end{tabular}




\section{Table 3}

Steroid dosing: idealized tapering schedule

\begin{tabular}{lc}
\hline Time & Dose (mg/kg/day) \\
\hline Intraoperative & $15-25$ \\
Postoperative day 1-6 & $3-10$ to $0.3-1$ \\
Weeks 2-3 & $0.25-0.75$ \\
Weeks 4-5 & 0.2 to $0.5-0.6$ \\
Weeks 6-7 & $0.17-0.2$ to $0.4-0.5$ \\
2 mo & 0.17 to $0.25-0.3$ \\
2.5 mo & 0.15 to $0.13-0.2$ \\
3 mo & $0.13-0.1$ \\
3.5 mo & $0.13-0.05$ \\
4 mo & $0.08-0$ \\
5 mo & $0.05-0$ \\
6 mo & 0 \\
\hline
\end{tabular}




\section{Table 4}

Demographics of steroid withdrawal

\begin{tabular}{lccc}
\hline \multicolumn{1}{l}{ OFF } & OFF $\rightarrow$ ON & ON \\
\hline $\mathrm{n}$ & $56(70 \%)$ & $18(22.5 \%)$ & $6(7.5 \%)$ \\
Recipient race & & & \\
$\quad$ Caucasian & $84 \%$ & $89 \%$ & $83 \%$ \\
$\quad$ Other & $16 \%$ & $11 \%$ & $17 \%$ \\
Mean recipient age (yr) & $10.3 \pm 5.6$ & $10.1 \pm 3.9$ & $13.3 \pm 3.8$ \\
Recipient sex & & & \\
$\quad$ Female & $29 \%$ & $39 \%$ & $67 \%$ \\
$\quad$ Male & $71 \%$ & $61 \%$ & $33 \%$ \\
Delayed graft function & & & \\
$\quad$ Yes & $4 \%$ & $0 \%$ & $17 \%$ \\
$\quad$ No & $96 \%$ & $100 \%$ & $83 \%$ \\
Mean recipient PRA & $5.7 \pm 15.3$ & $4.2 \pm 10.6$ & $34.5 \pm 38.6^{a}$ \\
Total HLA matches & $3.0 \pm 1.3$ & $2.3 \pm 1.2$ & $3.0 \pm 1.3$ \\
Total HLA mismatches & $2.8 \pm 1.4$ & $3.5 \pm 1.2$ & $2.8 \pm 1.3$ \\
Mean donor age (yr) & $27.3 \pm 15.2$ & $25.9 \pm 13.2$ & $39.3 \pm 5.8$ \\
Donor type & & & \\
Cadaveric & $54 \%$ & $67 \%$ & $67 \%$ \\
Living related & $46 \%$ & $33 \%$ & $33 \%$ \\
\hline
\end{tabular}

${ }^{a} P=0.0001$. 
Table 5

Time to steroid withdrawal and episodes of acute rejection ${ }^{a}$

\begin{tabular}{lccc}
\hline & OFF & OFF $\rightarrow$ ON & ON \\
\hline Time to steroid withdrawal (mo) (mean \pm SD) & $7 \pm 4$ & $9 \pm 6.5^{*}$ & \\
Time to restart steroids after withdrawal (mo) (mean \pm SD) & & $18 \pm 16$ & \\
Rejections & $39 \%$ & $77 \%$ & $100 \%^{* *}$ \\
$\quad$ Before withdrawal (\%) & & $100 \%$ & \\
$\quad$ After withdrawal (\%) & & & \\
\hline${ }^{*}{ }_{P=\mathrm{NS} ;}$ & & & \\
$* * *$ & &
\end{tabular}


Table 6

Evaluation at most recent follow-up ${ }^{a}$

\begin{tabular}{lcccc}
\hline & OFF & OFF $\rightarrow$ ON & ON & $\boldsymbol{P}$-value \\
\hline Creatinine $(\mathrm{mg} / \mathrm{dl})$ & $1.2 \pm 0.5$ & $1.8 \pm 0.9$ & 2 & 0.003 \\
Hematocrit $(\%)$ & $37.9 \pm 3.6$ & $34.7 \pm 4.4$ & 25.3 & NS \\
WBC $\left(1 \times 10^{3} / \mathrm{ml}\right)$ & $6.3 \pm 2.1$ & $6.7 \pm 1.9$ & 6.5 & NS \\
Platelets $\left(1 \times 10^{3} / \mathrm{ml}\right)$ & $203 \pm 56$ & $241 \pm 57$ & 175 & NS \\
Glucose $(\mathrm{mg} / \mathrm{dl})$ & $92 \pm 23$ & $97 \pm 23$ & 87 & NS \\
Cholesterol $(\mathrm{mg} / \mathrm{dl})$ & $160 \pm 28$ & $157 \pm 18$ & 130 & NS \\
Triglycerides $(\mathrm{mg} / \mathrm{dl})$ & $120 \pm 74$ & $128 \pm 58$ & 133 & NS \\
Systolic BP $(\mathrm{mmHg})$ & $112 \pm 16$ & $125 \pm 21$ & 140 & NS \\
Diastolic BP $(\mathrm{mmHg})$ & $73 \pm 11$ & $80 \pm 15$ & 80 & $\mathrm{NS}$ \\
Off antihypertensives & $77 \%$ & $44 \%$ & $0 \%$ & 0.003 \\
Tacrolimus level $(\mathrm{ng} / \mathrm{ml})$ & $6.4 \pm 2.4$ & $6.6 \pm 2.6$ & 13.3 & NS \\
\hline
\end{tabular}

${ }^{a} \mathrm{BP}$, blood pressure; WBC, white blood cells. 